\title{
Measurement and Interpretation of Lepton-Pair Production at LEP2 energies
}

\author{
Anette Behrmann \\ University of Wuppertal \\ DELPHI Collaboration, CERN \\ 12.08 .2000 \\ DPF2000, Electroweak Session V
}

- Introduction, Features of LEP2

- Measurement of Cross-Sections and $A_{f b}$

- Interpretations of the Results 


\section{Lepton-Pair Production at LEP2}
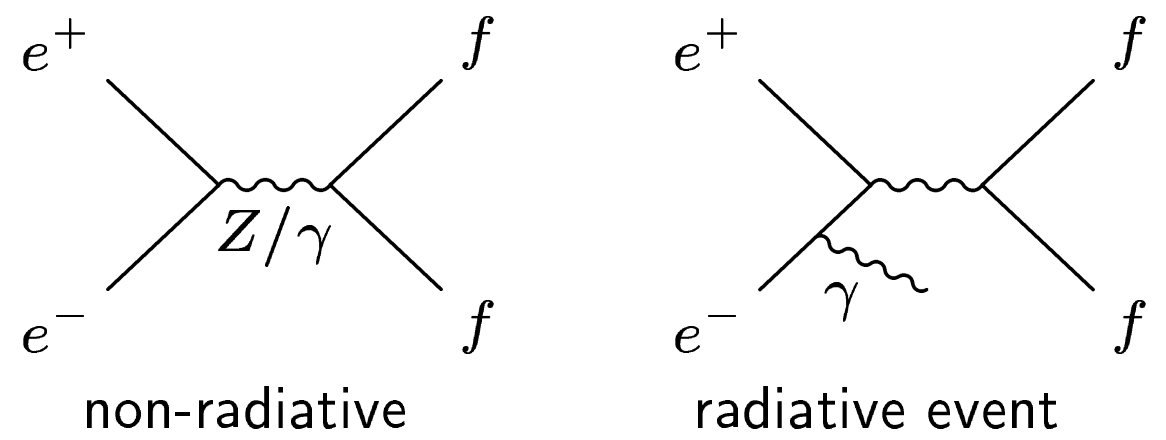

radiative event

- Motivation: Test of the Standard Model $\Rightarrow$ deviations may be a hint for new physics, indirect search

- observed channels: $f=e, \mu, \tau$

- at LEP2 many events undergo Initial State Radiation $\Rightarrow$ reduction of the effective centre-of-mass energy $\sqrt{s^{\prime}}$ $Z$ returns: $\sqrt{s^{\prime}} \approx M_{Z}$ (acollinear events)

$\sqrt{s^{\prime}}=m_{l \bar{l}}$, invariant Mass of the final Lepton-Pair after ISR and FSR (initial and final state radiation)

- calculation of cross-section and $A_{f b}$ for 2 event samples - inclusive sample (including radiative events)

- non-radiative sample

- non-radiative sample most sensitive to new physics effects 


\section{Determination of $\sqrt{s^{\prime}}$}

Computation of $\sqrt{s^{\prime}}$ using the following information:

- $E_{\text {Beam }}$

- directions and momentum of the final state leptons

- deposited energy of isolated photons in the calorimeters

\section{Angular Method}

$s^{\prime}=s-2 E_{\gamma} \sqrt{s}$ with energy of the ISR-Photon:

$$
E_{\gamma(I S R)}=\frac{\left|\sin \left(\theta_{1}+\theta_{2}\right)\right| \sqrt{s}}{\sin \theta_{1}+\sin \theta_{2}+\left|\sin \left(\theta_{1}+\theta_{2}\right)\right|}
$$

\section{Kinematic Fit}

(a) different topology assumptions $(\mu \mu, \mu \mu+\gamma, \ldots$.

(b) fitted parameters: $(|\vec{p}|, \theta, \phi)$

(c) constraints: energy and momentum conservation

(d) cut on $P\left(\chi^{2}\right)$

(e) $\sqrt{s^{\prime}}$ set accordingly to fitted parameters

$\Rightarrow$ improves the $\sqrt{s^{\prime}}$ resolution $\left(\Delta \sqrt{s^{\prime}} / \sqrt{s^{\prime}} \sim 5 \%\right)$ 


\section{Distributions of $\sqrt{s^{\prime}}$}
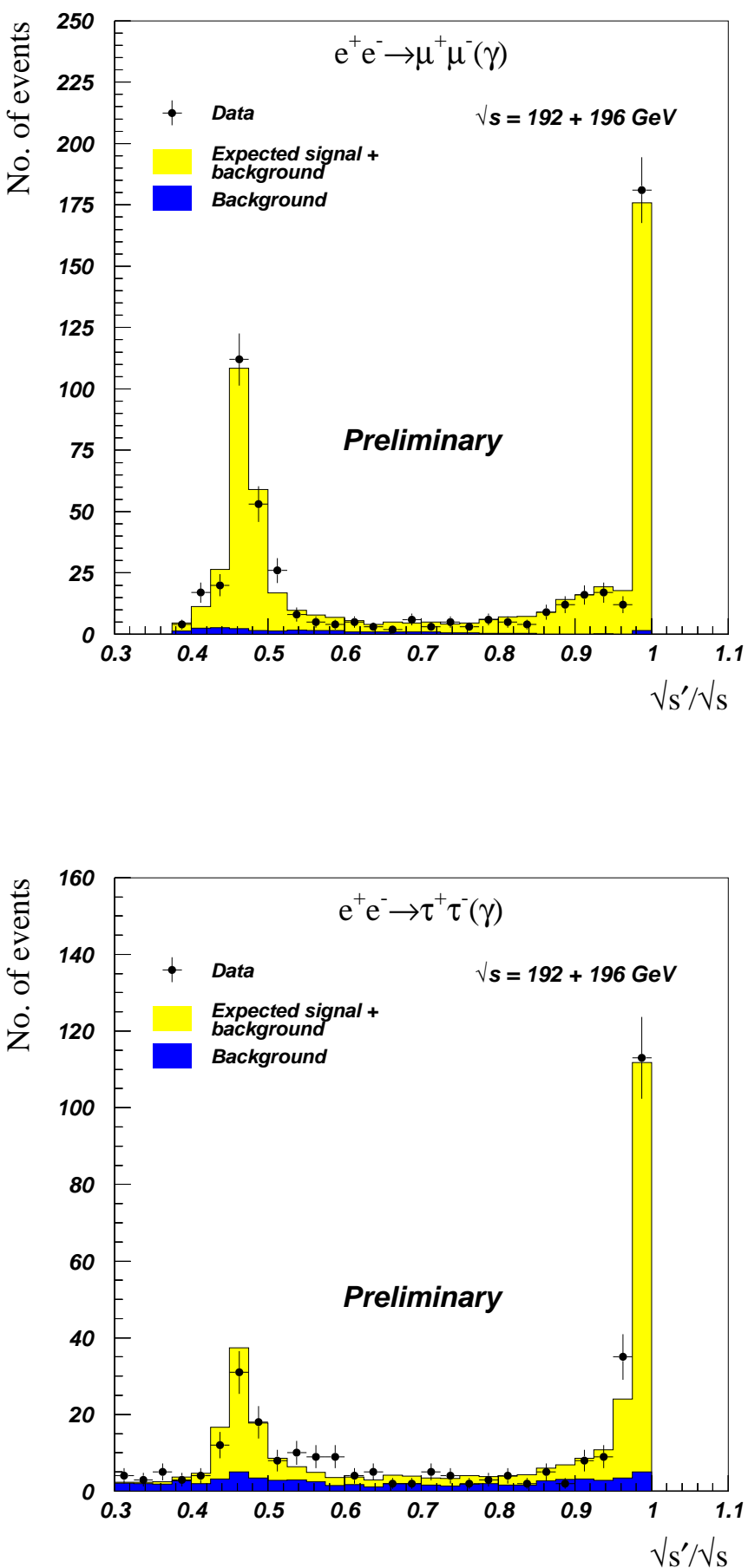

A.Behrmann, DPF 2000 in Columbus, Ohio, 9-13 August 2000 


\section{Event Selection}

\section{Different cuts for each channel on:}

- charged multiplicity

- impact to interaction point

- polarangle $(|\cos \theta| \leq 0.97 / 0.94$ for $\mu / \tau)$ $44^{\circ} \leq \theta \leq 136^{\circ}$ for electrons

- acollinearity

- inclusive class: $\sqrt{s^{\prime}}>75 \mathrm{GeV}(\mu, \tau)$

- non-radiative class $\sqrt{s^{\prime}} / \sqrt{s}>0.85(\mu, \tau)$ $\left(e^{+} e^{-} \rightarrow\right.$ acollinearity $\left.<20^{\circ}\right)$

$\underline{\text { Remaining Backgrounds: }}$

- 4-fermion-events (WW, ZZ, Ze $e^{+} e^{-}$, Weע...)

- 2-photon-events $\left(e^{+} e^{-} \rightarrow e^{+} e^{-} f \bar{f}\right)$

- Cosmics

- Feed through from radiative events
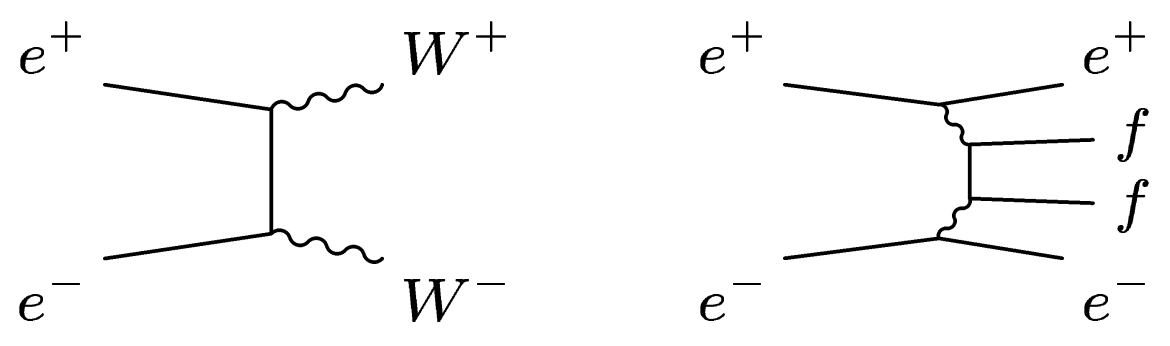


\section{Results for Lepton-Pair Selection}

\begin{tabular}{|c|c|c|c|c|c|}
\hline Year & 95 & 96 & 97 & 98 & 99 \\
\hline Energy $(\mathrm{GeV})$ & $130 / 136$ & $161 / 172$ & 183 & 189 & $192-202$ \\
\hline $\int \mathcal{L} d t(\mathrm{pb})^{-1}$ & $\sim 6$ & $\sim 20$ & $\sim 54$ & $\sim 155$ & $\sim 225$ \\
\hline $\begin{array}{c}\text { number of events } \\
e^{+} e^{-} \rightarrow e^{+} e^{-}(\gamma)\end{array}$ & $129 / 152$ & $276 / 312$ & 1109 & 2804 & 4446 \\
\hline$e^{+} e^{-} \rightarrow \mu^{+} \mu^{-}(\gamma)$ & $56 / 40$ & $72 / 70$ & 354 & 880 & 1256 \\
\hline$e^{+} e^{-} \rightarrow \tau^{+} \tau^{-}(\gamma)$ & $33 / 29$ & $58 / 54$ & 232 & 682 & 640 \\
\hline
\end{tabular}

Errors on $\sigma$ and $A_{f b}$ dominated by statistics

\section{DELPHI}

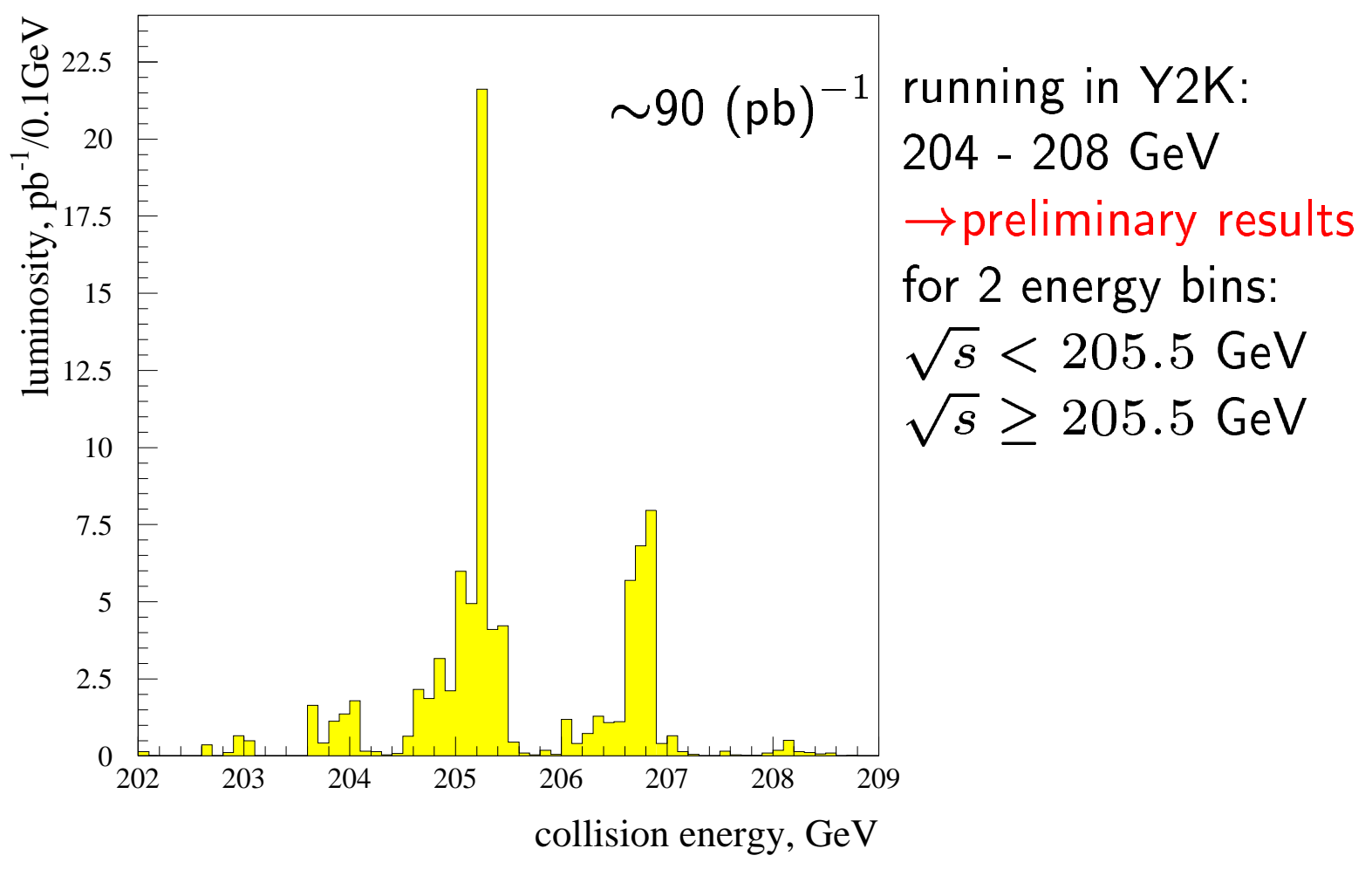




\section{Lepton Cross-Sections at LEP2}

\section{DELPHI}

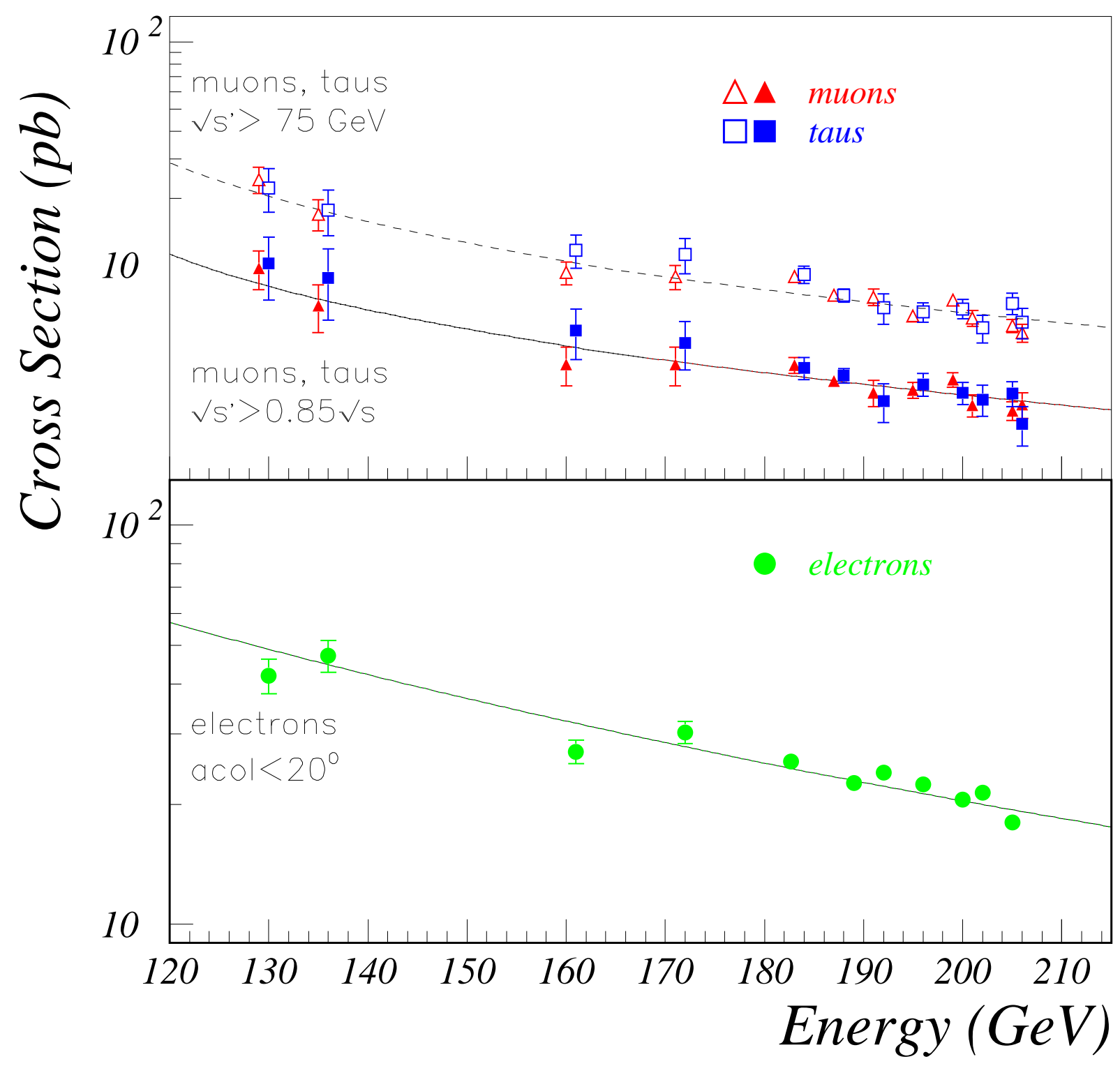

Typical Systematic Errors: $\mu \sim 3 \%, \tau \sim 5 \%, e \sim 1 \%$ 


\section{Lepton Asymmetries at LEP2}

\section{DELPHI}

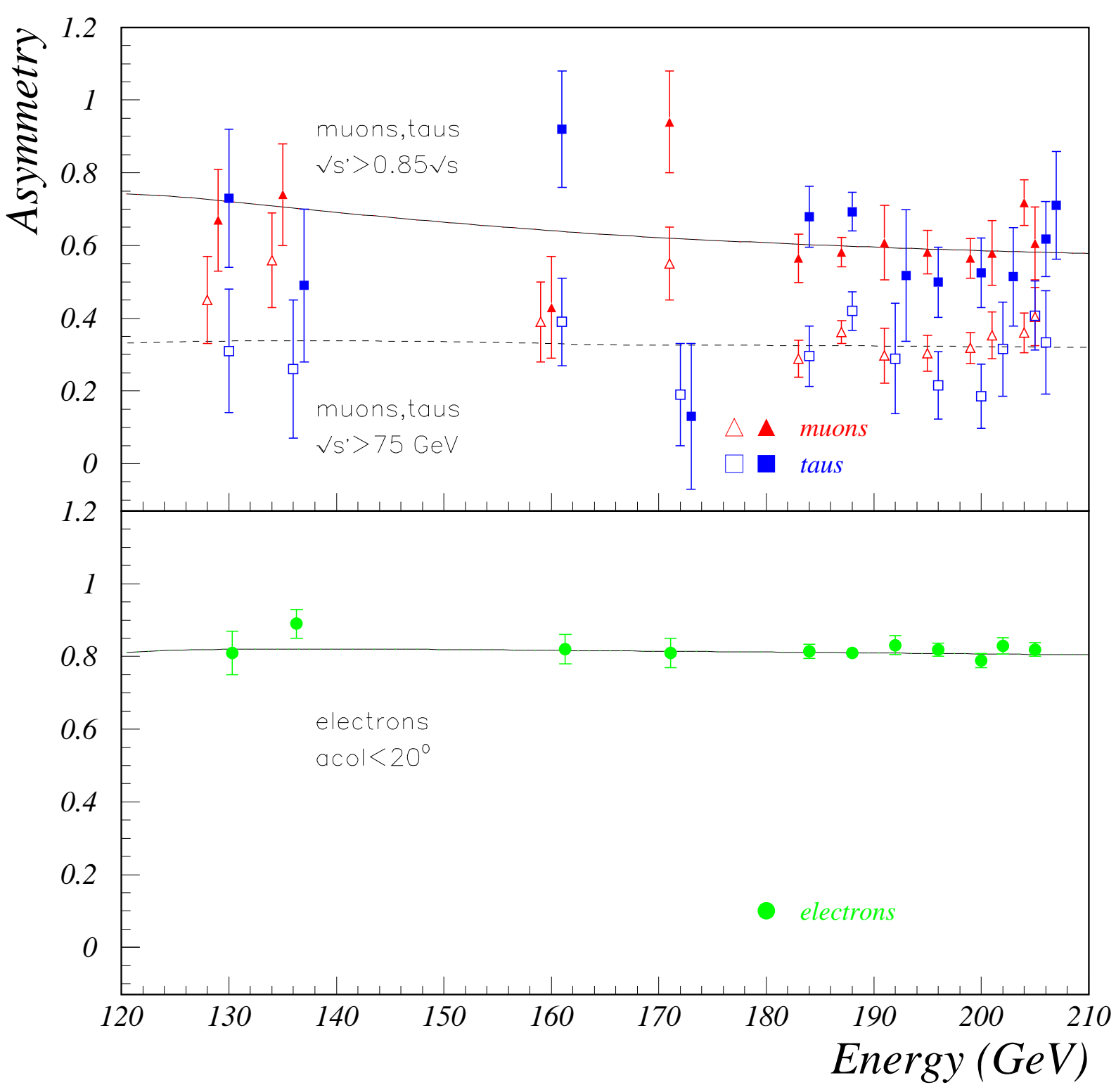

Typical Systematic Errors: $\mu \sim 10^{-3}, \tau \sim 10^{-2}, e \sim 10^{-3}$ All results in a reasonable agreement with the SM Prediction! 


\section{Differential Cross Sections}

\section{DELPHI PRELIMINARY}

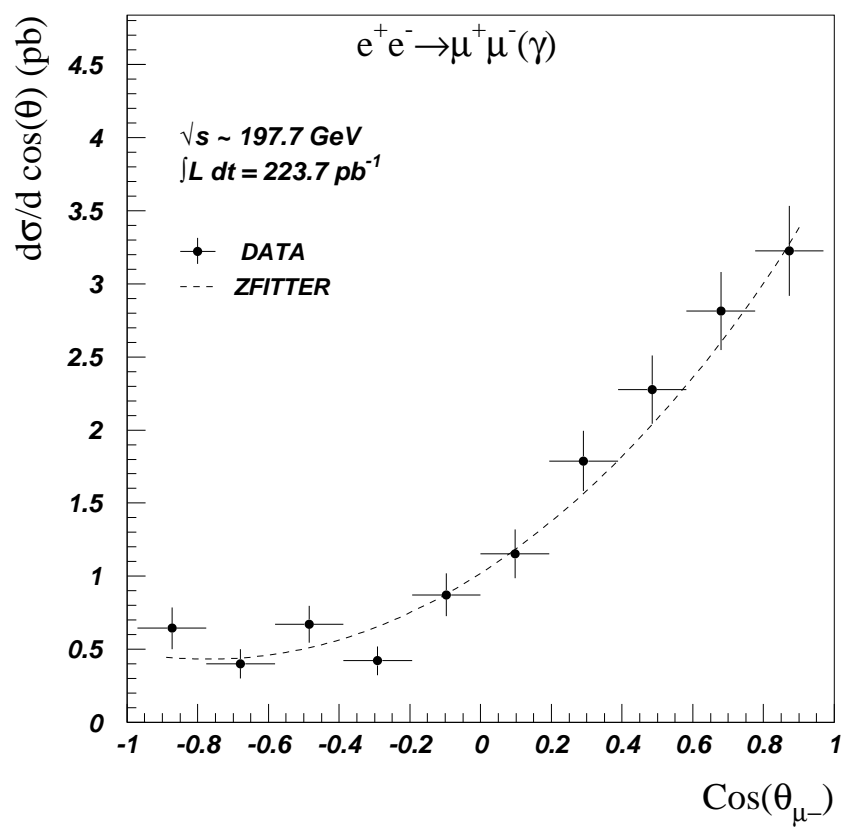

DELPHI PRELIMINARY

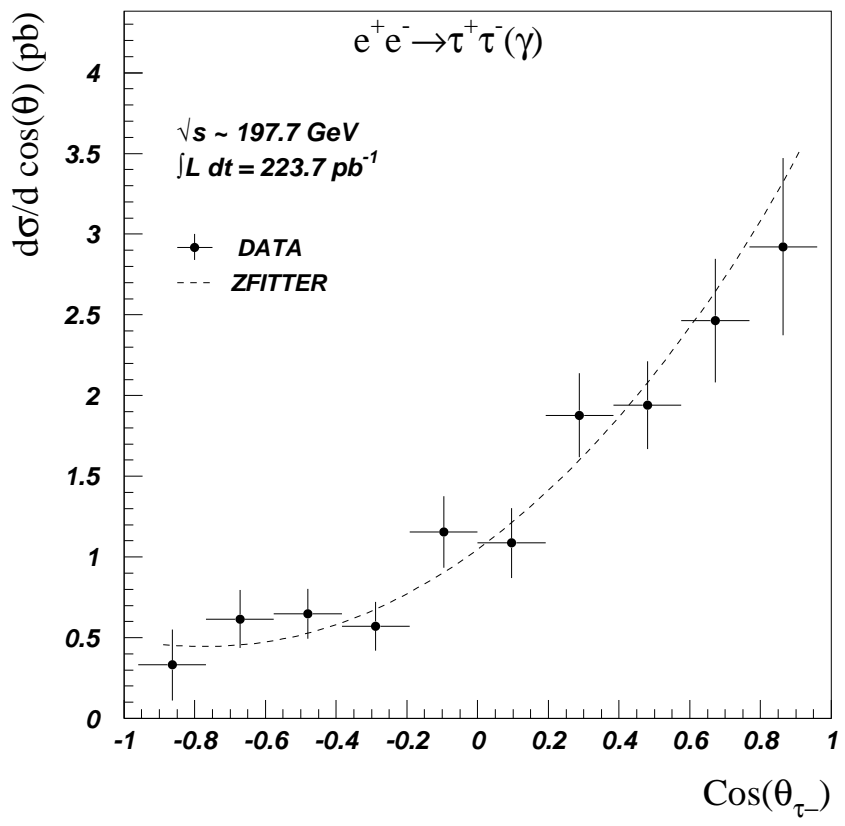

A.Behrmann, DPF 2000 in Columbus, Ohio, 9-13 August 2000 


\section{$Z^{\prime}$-Models}

Several theories predict an additional heavy gauge boson $Z^{\prime}$ parameters: mass $m_{Z^{\prime}}, Z Z^{\prime}$ mixing $\theta_{M}$, couplings to fermions $\Rightarrow$ Sensitivity above $Z$ resonanz: mass $m_{Z^{\prime}}$

Fits to measured $\sigma_{l l, q q}, A_{F B}(\mathrm{LEP} 1+2)$ with 2 different approaches:

- Model independent: $a_{f}^{\prime}, v_{f}^{\prime}$ free

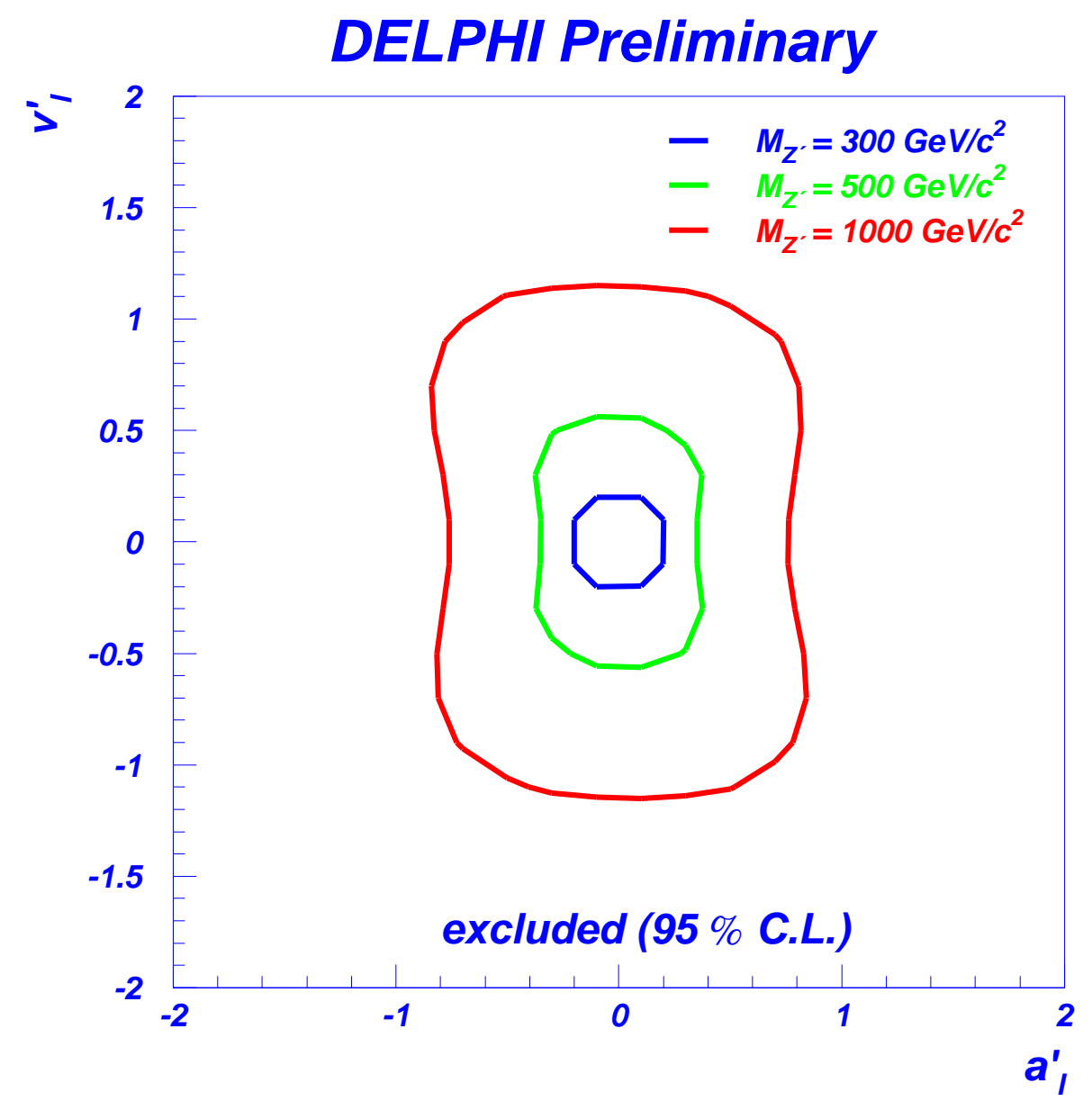




\section{$Z^{\prime}$-Models}

- Model dependent (E6-models, L-R model, sequential SM): $m_{Z^{\prime}}, \theta_{Z Z^{\prime}}$ free

\section{DELPHI Preliminary}

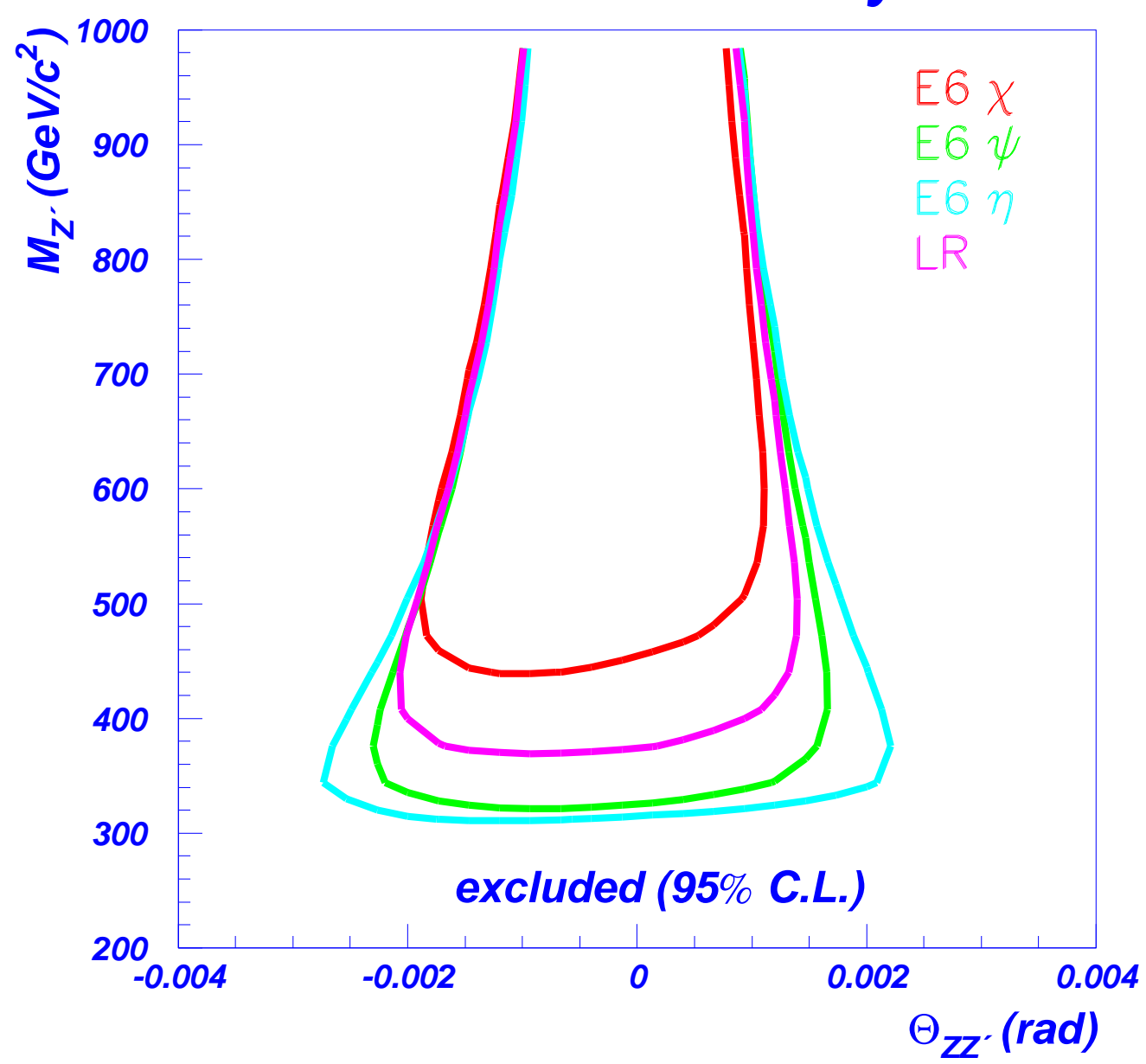

\begin{tabular}{|c|c|c|c|c|c|}
\hline Model & $\chi$ & $\psi$ & $\eta$ & L-R & SSM \\
\hline$m_{Z^{\prime}}^{\text {limit }}\left(\mathrm{GeV} / c^{2}\right)$ & 468 & 345 & 330 & 397 & 676 \\
\hline
\end{tabular}




\section{Contact Interactions}
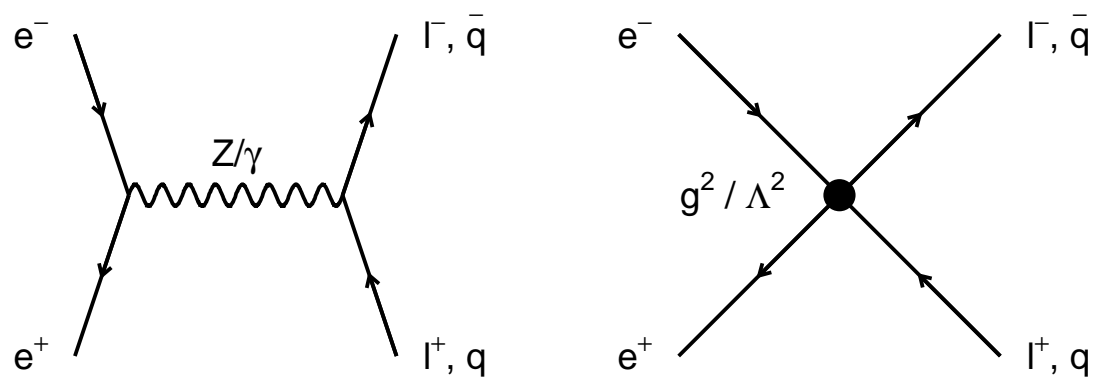

New physics with a high characteristic energy scale $(m \gg \sqrt{s})$ parameterized by the effective Lagrangian:

$\mathcal{L}_{e f f}=\frac{g^{2}}{(1+\delta) \Lambda^{2}} \sum_{i, j=L, R} \eta_{i j} \bar{e}_{i} \gamma_{\mu} e_{i} \bar{f}_{j} \gamma^{\mu} f_{j}$, assume $g^{2}=4 \pi$

- $\Lambda$ is the scale of C.I, $\epsilon=1 / \Lambda^{2}$ the fitted parameter,

- input: $\sigma_{l l}, A_{f b}$, assuming lepton universality

lower limits of the scale $\Lambda$ (at $95 \%$ C.L)

\section{DELPHI Preliminary}

$\Lambda^{-} \Lambda^{+}$

LR $\quad 6.9 \quad 9.7$

RL $\quad 6.9 \quad 9.7$

AA 13.710 .3

VV 12.719 .0

RR $\quad 7.8 \quad 9.6$

LL $\quad 8.010 .0$ $\epsilon$ compatible with

$\mathrm{SM} \epsilon=0$

at $2 \sigma$-level

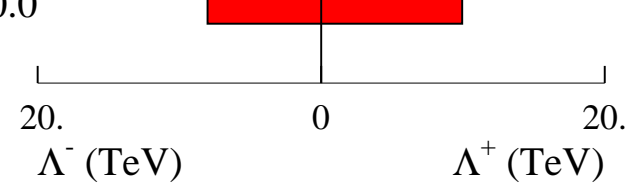




\section{Gravity in extra Dimensions}

- Process $e^{+} e^{-} \rightarrow G \rightarrow f \bar{f}$ $\frac{d \sigma}{d z}=A(z)+B(z)\left[\frac{\lambda}{M_{S}^{4}}\right]+C(z)\left[\frac{\lambda}{M_{S}^{4}}\right]^{2}, z=\cos (\theta)$

- parameter is $\epsilon=\frac{\lambda}{M_{S}^{4}}$

- fit the measured differential cross-sections $\left(\mu^{+} \mu^{-}, \tau^{+} \tau^{-}\right)$

- biggest effect at large $|\cos \theta|$

\section{DELPHI PRELIMINARY}

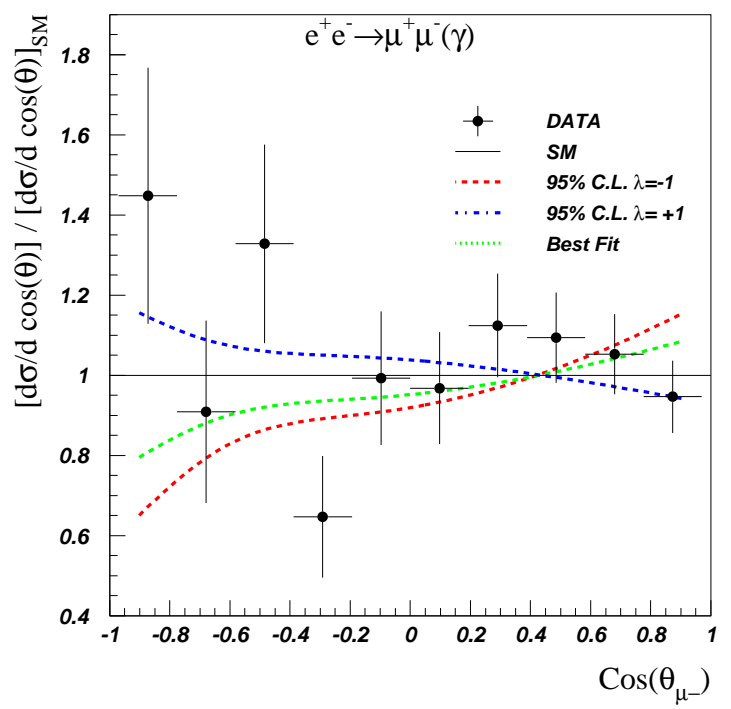

DELPHI PRELIMINARY

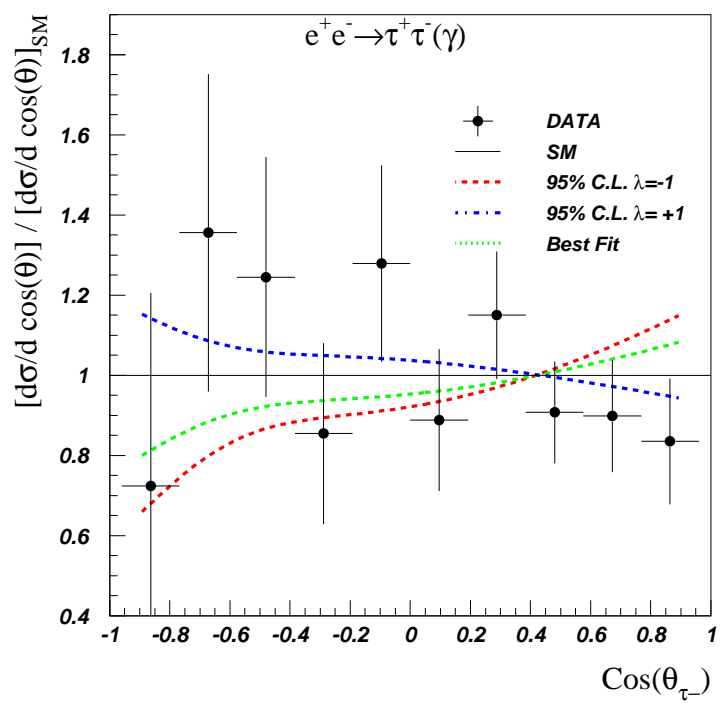

\begin{tabular}{|c|c|c|c|c|}
\hline Final State & $\sqrt{\mathrm{s}}(\mathrm{GeV})$ & $\epsilon_{\text {Best }}\left(\mathrm{TeV}^{-4}\right)$ & $\lambda$ & $\mathrm{M}_{\mathrm{S}}(\mathrm{TeV})$ \\
\hline \hline \multirow{2}{*}{$l^{+} l^{-}$} & $192-202$ & $-2.77_{-2.37}^{+2.39}$ & $\begin{array}{r}-1 \\
+1\end{array}$ & 0.623 \\
& & & 0.711 \\
\cline { 2 - 5 } & \multirow{2}{*}{$183-202$} & $-4.39_{-1.65}^{+2.10}$ & -1 & 0.598 \\
& & & 0.755 \\
\hline
\end{tabular}




\section{Conclusions}

- Lepton-pair production above the $Z$ resonanz still a good process to test the SM

- Results for the cross-sections and asymmetries at $130-202 \mathrm{GeV}$ in a good agreement with the SM expectations

- Preliminary results at $205-206 \mathrm{GeV}$ also consistent with the SM

- Interpretations in terms of possible new interactions gave no evidence for the existence of new physics, but limits on the energy scales ( $95 \%$ C.L.) were derived

(Z' boson > $350 \mathrm{GeV}$, Contact Interaction energy scale above 10-20 TeV, Gravity in extra Dimensions $M_{S}>600$ $\mathrm{GeV}$ )

- Last chance to find something new at LEP THIS YEAR! 